\title{
Las micorrizas arbúsculares: su valoración en el marco de la agroecología
}

Arbuscular mycorrhizae: their assessment in the framework of agroecology

\author{
Micorrizas arbusculares: sua avaliação no âmbito da agroecología
}

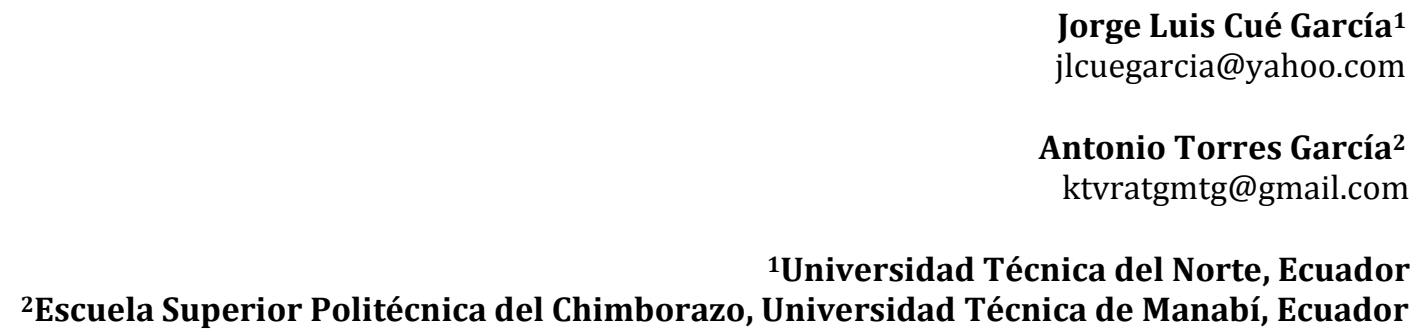

Artículo recibido enero 2019, arbitrado mayo 2019 y publicado en septiembre 2019

\section{RESUMEN}

En el presente artículo se realiza una valoración de las micorrizas en el marco de la agroecología mediante una revisión de resultados de experiencias en diversas latitudes. La agroecología propone un enfoque integral y sustentable de los sistemas de producción agrícola, así entonces, las micorrizas arbúsculares definida como asociaciones ecológicamente mutualistas entre hongos del Phyllum Glomeromycota y la inmensa mayoría de las plantas, se entiende como una alternativa útil para una agricultura sustentable. Contradictoriamente su empleo en la práctica agrícola no posee una amplia representación en la generalidad de los países de América Latina, aun cuando se reconocen los múltiples efectos positivos de las micorrizas arbúsculares en las plantas, el suelo y el medio ambiente en general.

Palabras clave: micorrizas; agroecoogia, sutentabilidad

\begin{abstract}
In this article, an assessment of mycorrhizae in the framework of agroecology is made through a review of the results of experiences in various latitudes. Agroecology proposes a comprehensive and sustainable approach to agricultural production systems, thus, arbuscular mycorrhiza defined as ecologically mutualistic associations between Phyllum Glomeromycota fungi and the vast majority of plants, is understood as a useful alternative for sustainable agriculture. Contradictorily, its use in agricultural practice is not widely represented in the majority of Latin American countries, even though the multiple positive effects of arbuscular mycorrhizae on plants, soil and the environment in general are recognized.
\end{abstract}

Key words: mycorrhizae; agroecoogy, sustainability 


\section{RESUMO}

Neste artigo, uma avaliação das micorrizas no âmbito da agroecologia é feita através de uma revisão dos resultados de experiências em várias latitudes. A agroecologia propõe uma abordagem abrangente e sustentável dos sistemas de produção agrícola, portanto, micorriza arbuscular definida como associações ecologicamente mutualísticas entre os fungos Phyllum Glomeromycota e a grande maioria das plantas, é entendida como uma alternativa útil para a agricultura sustentável. Contraditoriamente, seu uso na prática agrícola não está amplamente representado na maioria dos países da América Latina, embora sejam reconhecidos os múltiplos efeitos positivos das micorrizas arbusculares nas plantas, no solo e no ambiente em geral.

Palavras-chave: micorrizas; agroecoogia, sustentabilidade

\section{INTRODUCCIÓN}

En la Constitución de la República del Ecuador 2008 se establece en su artículo 14 que "se reconoce el derecho de la población a vivir en un ambiente sano y ecológicamente equilibrado, que garantice la sostenibilidad y el buen vivir, Sumak Kawsay. Se declara de interés público la preservación del ambiente, la conservación de los ecosistemas, la biodiversidad y la integridad del patrimonio genético del país, la prevención del daño ambiental y la recuperación de los espacios naturales degradados". Así también en su artículo 281 plantea que "la soberanía alimentaria constituye un objetivo estratégico y una obligación del Estado para garantizar que las personas, comunidades, pueblos $\mathrm{y}$ nacionalidades alcancen la autosuficiencia de alimentos sanos y culturalmente apropiado de forma permanente". Destaca en su numeral 3 "fortalecer la diversificación y la introducción de tecnologías ecológicas y orgánicas en la producción agropecuaria" ( $p$. 138), en tanto el numeral 8 se establece "asegurar el desarrollo de la investigación científica y de la innovación tecnológica apropiadas para garantizar la soberanía alimentaria".

En el Plan Nacional para el Buen Vivir 2013-2017, regido por la Secretaria Nacional de Planificación y Desarrollo, define que "el Estado, mediante una política ambiental sostenida, garantizará la conservación o gestión sustentable de los ecosistemas del país, de sus funciones, de las especies y poblaciones nativas y de la agrobiodiversidad". En este mismo documento se proyecta en la agenda resumen para la Zona 1: provincias de Carchi, Esmeraldas, Imbabura y Sucumbíos, en el marco de la Transformación de la matriz productiva, dentro de las principales líneas de acción "desarrollar de manera sustentable el sector agropecuario y pesquero fomentando los encadenamientos productivos articulados a la economía popular y solidaria de la zona".

Dentro de las múltiples alternativas agroecológicas que se pueden emplear en los sistemas de producción agrícolas de cultivos, se encuentran las micorrizas vesículos arbúsculares, biofertilizante de comprobadas bondades para las plantas, la rizosfera de estas, el suelo y el medio ambiente en general.

Un gran número de especies de plantas, que se constituyen en cultivos de interés agrícola, son capaces de establecer asociaciones ecológicamente mutualistas con hongos que forman arbúsculos al interior de las células de las plantas. Esta estructura, los arbúsculos, es donde se realiza el intercambio de carnono y fósforo entre el hongo y la planta. A tipo de simbiosis hongo-planta se le reconoce como micorrizas arbúsculares. 
Las micorrizas arbusculares (MA) son asociaciones ecológicamente mutualistas que se establecen entre un selecto grupo de hongos (Glomeromycota) y la gran mayoría de las plantas. Aproximadamente un $80 \%$ de las familias de plantas existentes tienen la potencialidad de formar este tipo de asociación (Trappe, 1987). Las MA son el tipo de micorrizas que forman la mayoría de las plantas de interés agrícola. En dicha asociación, el hongo forma arbúsculos que son las estructuras donde se realiza el intercambio de carbono y fósforo entre el hongo y la planta. Algunos hongos micorrízicos forman vesículas en el micelio interno, las cuales son estructuras de reserva del hongo.

En el presente trabajo se abordarán algunas consideraciones acerca de las micorrizas arbúsculares en el marco de la agroecología.

\section{METODOLOGÍA}

Se basó en una revisión documental, en donde se tomaron como principales fuentes documentales plataformas de búsqueda en línea, como google Académico, Scielo, Scopus, Latindex, Eric, entre otras fuentes de recolección de información, se compilaron más de cuarenta estudios asociados a la micorrizas arbúsculares, en donde se descartaron estudios que no valoraban métodos de sustentabilidad y la preservación del ambiente, también se consideraron un grupo de veintiún, trabajos en donde se realiza el principal análisis de la valoración que se hace acerca de la agroecología en las micorrizas arbúsculares.

\section{DISCUSIÓN}

\section{Bondades de la micorrización para las plantas}

Resulta evidente que los hongos micorrizogenos han coevolucionado con las plantas por más de 400 millones años, Honrubia (2009), logrando establecerse en una amplia diversidad de medios naturales del planeta.

En una reciente, y muy completa, revisión sobre la distribución filogenética y evolución de las micorrizas en las plantas terrestres, Wang y Qiu (2006) confirman que el 80 y $92 \%$, respectivamente de las especies $y$ familias de plantas testadas, son micorrícicas, y que son precisamente las micorrizas arbúsculares las predominantes, estando además presentes entre las plantas más primitivas, como hepáticas, pteridófitos y las gimnospermas más antiguas. (Honrubia, 2009).

Las expectativas de investigación de la simbiosis micorrizas-plantas siguen siendo muy amplias según Clemmensen y otros (2013), destacando que recientemente se ha puesto énfasis en una perspectiva multifuncional más amplia, incluyendo los efectos de la simbiosis micorrícica sobre las comunidades vegetales y microbianas, así como en los procesos del ecosistema. Es relevante la movilización de $\mathrm{N}$ y $\mathrm{P}$ a partir de polímeros orgánicos, la liberación de los nutrientes de las partículas minerales, los efectos sobre el ciclo del carbono, la mediación de la respuesta de las plantas a factores de estrés como la sequía, la acidificación del suelo, metales tóxicos y patógenos de plantas, así como una amplia gama de posibles interacciones con grupos de otros microorganismos del suelo.

En la compleja interacción planta-hongo micorrícico existe un tercer asociado que pertenece a las bacterias. El establecimiento de esta triada asociativa, Bonfante y Anca (2009) plantean que se propicia a partir de la liberación de moléculas activas, incluyendo compuestos volátiles, y el contacto físico entre los socios. 
Se ha demostrado que los asimilados pueden ser transportados de una planta a otra a través de las conexiones de hifas de los hongos micorrizógenos (Mohammadi, Khalesro, Sohrabi y Heidari, 2011).

La interacción de las plantas y los hongos micorrícicos arbúsculares es de tipo mutualistas y sus efectos son en general positivos para las plantas en cuanto a:

Estado nutricional [Heikham, Rupam y Bhoopander, (2009); Felderer, Jansa y Schulin (2013); Li, Guan, Stonor, Smith Y Smith, (2013)].

Estado fitosanitario, [Vannette, Hunter y Rasmann (2013); Vos, y Otros, (2013); Liu, Li, y Tian (2013) Ahmed, Sallam, Mohamed y Hassan (2013); Zhipeng, Fayolle, Van Tuinen... Gianinazzi (2012); Jung, Martínez, López y Pozo (2012) y Pivato, Offre, Marchelli... Berta (2009)] producción de biomasas fresca y seca, [Miransari, y Otros, (2013)].

Adaptación a condiciones de estrés por factores abióticos tales como: baja disponibilidad de fósforo, salinidad $\mathrm{y} / \mathrm{o}$ presencia de metales pesados en el suelo; variaciones de la temperatura fuera del rango ecológico de los cultivos y/o condiciones de sequía. [Watts-Williams, Patti, y Cavagnaro (2013); Li, et al., (2013); Heikham et al., (2009) y Mohammadir et al., (2011)].

La ocurrencia de la micorrización permite, en criterio de Garg y Manchanda (2008), el aumento de la nodulación y la fijación de nitrógeno en las leguminosas, favoreciendo una mejor nutrición con fósforo en las plantas inoculadas que mejoran su tasa de crecimiento y aumentan la producción de antioxidantes.

Las razones más aceptadas para la no proliferación de la simbiosis micorrícicas, cuando se realizan aplicaciones de altas dosis de fósforo, según Douds (s/f) son las siguientes:

- Las raíces, en ambientes de alto fósforo, no exudan tantos productos estimulantes de ramificaciones de hifa y por eso hay menos contactos de infección o colonización.

- Plantas con mayor fósforo no proporcionan tantos carbohidratos al hongo. Esto limita la colonización desde la raíz al suelo.

- Menos carbohidratos suplidos al hongo reducen la cantidad de esporulación por falta de energía adecuada.

\section{Bondades de la micorrización para el suelo}

En relación al suelo, los hongos micorrizogenos constituyen un componente vital de su microbiología, pues se reconoce que producen la glomalina, una glicoproteína protectora de la hifa, tiene efecto de aglutinante natural que estimula la agregación de partículas en el suelo. La glomalina que ha demostrado gran persistencia en el suelo y capacidad de ocupar una fracción significativa de la materia orgánica y carbono total del suelo. (Douds, s/f.). 


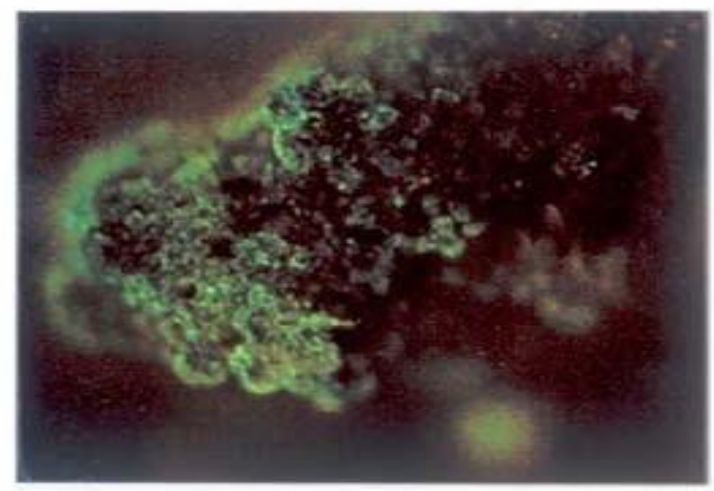

Glomalin is the green material on this soil aggregate.

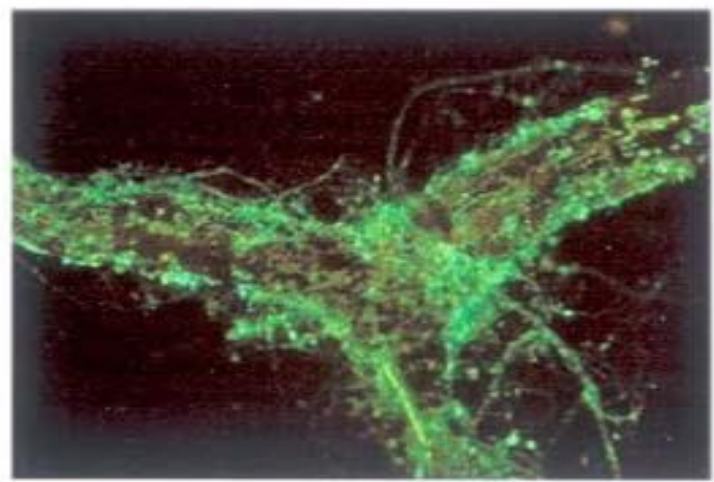

An arbuscular mycorrhizal fungus colonizing a root. Hyphae are the thread-like filaments. The

Figura 1. El papel de los hongos formadores de micorrizas y su manejo. (Douds, s/f).

El efecto benéfico de la simbiosis hongoplanta sobre el incremento de la extensión y volumen del sistema de raíces, así como de las hifas de los hongos, permiten una mayor retención física de las partículas de suelo.

La interacción de la simbiosis micorricica con otros microorganismos del suelo posibilita el establecimiento de cooperaciones beneficiosas con una amplia representación de estos y compitiendo con otros, que generalmente son de tipo fitopatógeno.

Para la biorremediación de suelos, el aumento de la biomasa de las plantas, es la razón más importante de su eficacia. Por tanto, si las plantas que logran la micorrización producen mayor cantidad de biomasa seca de manera significativa según Zaefarian, Rezvani, Ardakani, Rejali y Miransari, (2013), entonces la inoculación de plantas con hongos micorrizogenos $\mathrm{y}$ estimular la comunidad natural de micorrizas nativas del suelo en las condiciones de los sistemas de producción agrícola, se constituyen en alternativas para la biorremediación de suelos.

\section{Algunas prácticas agroecológicas para favorecer la micorrización}

En tanto persiste el modelo convencional de producción agrícola denominado "revolución verde", el cual se basa en el uso intensivo de energía, maquinaria y sustancias químicas de origen sintético, se hace necesario destacar que desde hace aproximadamente 50 años se le ha contrapuesto el pensamiento de ecologistas y los movimientos que apoyan esta tendencia. Así también ha aparecido en los medios académicos el término de agroecología, como una propuesta de alternativas amigables con el medio ambiente, que en la actualidad ya demuestra resultados consolidados en la práctica social.

De manera general se sugieren algunas alternativas agroecológicas que tienden a favorecer la micorrización, mismas que se corresponden de manera significativa con algunas de las recomendadas para la conservación de suelos y agua.

Labranza mínima del suelo, tanto en acondicionamiento y preparación como en la atención a los cultivos son:

- Rotación, asociación, sucesión de cultivos.

- Aportaciones de fuentes orgánicas al suelo.

- Mantener el suelo cubierto, en alternativas de cobertura muerta y/o viva.

- No quema de los residuos de cosecha. 
Manejo de cuantías mínimas en la dosificación de los fertilizantes químicos y de los pesticidas, en particular el fósforo $\mathrm{y}$ fungicidas, respectivamente. En los casos posibles prescindir de su uso.

En opinión de Cuenca, y Otros, (2007) “en muchos países latinoamericanos la agricultura carece actualmente de una eficiente extensión técnica, lo que ha conducido al uso indiscriminado de agroquímicos y fertilizantes. Ello hace necesario desarrollar y divulgar estrategias de manejo agrícola que permitan minimizar estos problemas".

\section{A MANERA DE CONCLUSIÓN}

Las micorrizas arbúsculares se constituyen en una alternativa ecológica y agronómicamente factible para diversos sistemas de producción agrícola, en tanto muestran una alta plasticidad ecológica, se manifiesta en la mayoría de los cultivo de interés agrícola y existen los fundamentos científico y técnicos para su implementación en la práctica social.

\section{REFERENCIAS}

Ahmed, M. S., Sallam, N. M., Mohamed, A. A., y Hassan, M H. Archives of Phytopathology \& Plant Protection. May 2013; Vol. 46 Issue 7, p868-881. 14p. ISSN: 0323-5408 doi: $\quad 10.1080 / 03235408.2012 .753707$ [fecha de consulta: 12 febrero 2014].

Asamblea Nacional de la República del Ecuador. (2011). Constitución de la República del Ecuador 2008. [En línea] Última modificación: 13-jul-2011. [fecha de consulta: 22 noviembre 2013]. Disponible en: http://www.oas.org/juridico/PDFs/mesic ic4_ecu_const.pdf.

Bonfante, P., Anca I. A. (2009). Plants, mycorrhizal fungi, and bacteria: a network of interactions. Annual Review Microbiology ; 63:363-83.

Clemmensen, KE, Bahr, A., Ovaskainen, O., Dahlberg, A., Ekblad, A., Wallander, H.,... y
Lindahl, BD (2013). Las raíces y los hongos asociados conducen el secuestro de carbono a largo plazo en los bosques boreales. Science, 339 (6127), 1615-1618.

Cuenca G., Cáceres A., Oirdobro G., Hasmy Z., Urdaneta C. (2007). Las micorrizas arbusculares como alternativa para una agricultura sustentable en areas tropicales. INCI [revista en la Internet]. ; 32(1): 23-29. Disponible en: http://www.scielo.org.ve/scielo.php?scrip $\mathrm{t}=$ sci_arttext\&pid=S03781844200700010 0006\&lng=es.

Douds, D. (s/f.). El papel de los hongos formadores de micorrizas y su manejo. Disponible en: http://www.produccion animal.com.ar/produccion_organica_y_tra zabilidad/45micorrizas.pdf.

Felderer, B., Jansa, J., y Schulin, R. (2013). Interaction between root growth allocation and mycorrhizal fungi in soil with patchy $\mathrm{P}$ distribution. Plant \& Soil, 373(1/2), 569-582. doi:10.1007/s11104013- 1818-6 [Consulta:12 febrero 2018]. Disponible

en:http://web.ebscohost.com/ehost/detai l?sid=f31762ce-b8ef-4695-a762-

02a301d0c826\%40sessionmgr115\&vid=1 \&hid=112\&bdata=JnNpdGU9ZWhvc3QtbG l2ZQ\%3d\%3 d\#db=a9h\&AN=92041578.

Heikham, E., Rupam K., y Bhoopander, G. (2009). Arbuscular mycorrhizal fungi in alleviation of salt stress: a revie. En: Annals of Botany.; 104(7):1263-1280 [fecha de consulta: 15 noviembre 2018]. Disponible en:http://aob.oxfordjournals.org/content /104/7/1263.abstract.

Honrubia M. (2009). Las micorrizas: una relación planta-hongo que dura más de 400 millones de años. Anales del Jardín Botánico de Madrid Vol. 66S1: 133-144, ISSN: 0211-1322 doi: 10.3989/ajbm.2226

Jung, S.C, Martinez Medina, A., Lopez Raez J.A., Pozo M.J. (2012). Mycorrhiza-induced resistance and priming of plant defenses. Journal Chemical Ecology; 38(6):651-64. [Consulta: 22 noviembre 2018]. Disponible en: http://www.ncbi.nlm.nih. gov/pubmed/22623151.

Li, A., Guan, K., Stonor, R., Smith, S. E., y Smith, F. (2013). Direct and indirect influences of 
arbuscular mycorrhizal fungi on phosphorus uptake by two root hemiparasitic Pedicularis species: do the fungal partners matter at low colonization levels? Annals Of Botany; 112(6), 10891098. [Consulta: 12 febrero 2018] Disponible en: http://web.ebscohost.com /ehost/detail?sid=5f4f9964-32ca-4412be5e4e3050288807\%40sessionmgr111\& vid $=1 \&$ hid $=112 \&$ bdata $=J n N p d G U 9 Z W h v c$ 3QtbGl2ZQ\%3d\%3d\#db=a9h\&AN=90504 687

Liu, Z. L., Li, Y. J., Hou, H. Y., Zhu, X. C., Rai, V., He, X. Y., y Tian, C. J. (2013). Differences in the arbuscular mycorrhizal fungiimproved rice resistance to low temperature at two $\mathrm{N}$ levels: aspects of $\mathrm{N}$ and $\mathrm{C}$ metabolism on the plant side. Plant Physiology and Biochemistry, 71, 87-95. Available from: Academic Search Complete, Ipswich, MA. [Consulta: 14 enero 2018].

Miransari, M. M., Maleki, T., Besahrati, H. H., Rasuli Sadaghiani, M. H., y Tavassoli, A. A. (2013). Using pseudomonas spp. And arbuscular mycorrhizal fungi to alleviate the stress of zinc pollution by corn (Zea mays L.) Plant.Journal Of Plant Nutrition; 36(13), 2061-2069. doi:10.1080/ 01904167.2013.81673. [Consulta: 12 febrero 2018]

Mohammadi, K.; Khalesro, S.; Sohrabi, Y. y Heidari, G. A. (2018). Review: Beneficial Effects of the Mycorrhizal Fungi for Plant Growth. Journal of Applied Environmental and Biological Sciences, 2011; 1(9):310319., ISSN: 2090-4215. [Consulta: 22 noviembre]. Disponible en: http://www.textroad.com/pdf/JAEBS/J.\% 20Appl.\%20Environ.\%20Biol.\%20Sci.,\%2 01(9)30- 319,\%202011.pdf.

Pivato, B, Offre, P., Marchelli, S., Barbonaglia, B., Mougel, C, Lemanceau, P., Berta, G. (2009). Bacterial effects on arbuscular mycorrhizal fungi and mycorrhiza development as influenced by the bacteria, fungi, and host plant. Mycorrhiza. ; 19(2):81-90. doi: 10.1007/s00572-008-0205-2. [Consulta 15 noviembre 2018]. Disponible en: http://www.ncbi.nlm.nih.gov/pubmed/189 41805
Secretaria Nacional de Planificación y Desarrollo. Plan Nacional para el Buen Vivir 2013-2017. Quito, Ecuador. 2013. $594 \mathrm{p}$.

Trappe, J. M. (1987). Phylogenetic and ecologic aspects of mycotrophy in the angiosperms from an evolutionary standpoint. Ecophysiology of VA mycorrhizal plants, 5-25.

Vannette, RL, Hunter MD and Rasmann S. (2013). Arbuscular mycorrhizal fungi alter above- and below- ground chemical defense expression differentially among Asclepias species. Front. Plant Sci. 4:361. doi:10.3389/fpls.2013.00361-See more at: http://journal.frontiersin.org/Journal/10. 3389/fpls.2013.00361/abstract\#sthash.a ONrTcSW.dpuf [Consulta: 12 febrero 2018]. Disponible en: http://www.frontiersin.org/plantmicrobe_interaction/10.3389/fpls.2013.0 0361/abstract.

Vos, C., Schouteden, N., Van Tuinen, D., Chatagnier, O., Elsen, A., De Waele, D., Panis, B., y Gianinazzi-Pearson, V. Mycorrhiza-induced resistance against the root-knot nematode Meloidogyne incognita involves priming of defense gene responses in tomato. Soil Biology \& Biochemistry. 2013; Vol. 60, p45-54. 10p. doi:10.1016/j.soilbio.2013.01.013

[Consulta: 12 febrero 2018]

Watts-Williams, S., Patti, A., Cavagnaro, T. (2013). Arbuscular mycorrhizas are beneficial under both deficient and toxic soil zinc conditions. Plant \& Soil. Oct., Vol. 371 Issue 1/2, p299-312. 14p. 3 Charts. Doi: $\quad$ 10.1007/s11104-013-1670-8. [Consulta: 31 enero 2014]. Disponible en: http://web.b.ebscohost.com/ehost/detail ?sid=415371fc-c134-4e78-93ae-

9ec2a5319bdb\%40sessionmgr110\&vid=1 \&hid=119\&bdata=JnNpdGU9ZWhvc3QtbG $12 Z Q \% 3 d \% 3 d \# d b=a 9 h \& A N=90428689$.

Zaefarian, F., Rezvani M., Ardakani M., Rejali F. $y$ Miransari M. (2013). Impact of Mycorrhizae Formation on the Phosphorus and Heavy-Metal Uptake of Alfalfa, Communications in Soil Science and Plant Analysis. 44:8, 1340-1352, DOI:10.1080/00103624.2012.756505. 
Zhipeng, H., Fayolle l., Van Tuinen D., Chatagnier, O.,1, Li, X., Gianinazzi S., y Gianinazzi-Pearson, V. (2012). Local and systemic mycorrhiza-induced protection against the ectoparasitic nematode Xiphinema index involves priming of defence gene responses in grapevine.
Journal of Experimental Botany; 63(10):3657-3672,

doi:10.1093/jxb/ers046. [Consulta: 15 noviembre 2018]. Disponible en: http://jxb.oxfordjournals.org/open_acces s.html 